\title{
Chronische Schmerzen bei den Jüngsten verhindern
}

Fast die Hälfte aller Kinder und Jugendlichen klagt über Schmerzen. Damit diese nicht chronisch werden, brauche es maßgeschneiderte Präventionsmaßnahmen und bessere Versorgungsangebote für die Jüngsten, fordert die Österreichische Schmerzgesellschaft.

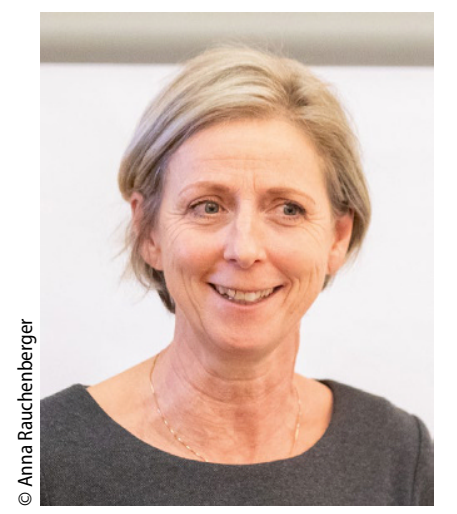

$\Delta$ OÄ Dr. Manuela Platzer, Klagenfurt

Eine deutsche Studie zur Gesundheit von Kindern und Jugendlichen zeigt, dass $47 \%$ der Befragten über wiederkehrende Schmerzen klagen. Rund fünf Prozent der Kinder sind im Alltag durch Schmerzen wesentlich beeinträchtigt. Für Österreich gibt es zwar keine entsprechenden Erhebungen, Expert*innen gehen aber davon aus, dass die Lage hierzulande ähnlich alarmierend ist.

Chronische, das heißt länger als drei Monate anhaltende Schmerzen im Kindesalter beeinträchtigen nicht nur die Lebensqualität der Betroffenen, sie ziehen oft weiterer Leiden nach sich: Junge Schmerzpatient ${ }^{*}$ innen laufen Gefahr, Ängste oder Schlafstörungen zu entwickeln und haben ein erhöhtes Risiko für chronische Beschwerden und eine gröBere Schmerzempfindlichkeit im Erwachsenenalter. Zudem kommt es zu vielen Schulfehltagen, und es droht soziale Isolation.

„Die Ursachen chronischer Schmerzen bei Kindern sind vielschichtig und lassen sich nicht auf rein körperliche oder seelische Faktoren reduzieren“, erklärt OÄ Dr. Manuela Platzer, Abteilung für Anästhesiologie, allgemeine Intensivmedizin, Notfallmedizin, interdisziplinäre Schmerzmedizin und Palliativmedizin, Klinikum Klagenfurt am Wörthersee. Die Behandlung und Vorbeugung chronischer Schmerzen benötigt deshalb auch eine multimodale interdisziplinäre Schmerztherapie, die neben der medikamentösen Versorgung auch psychosoziale Interventionen beinhaltet.

Für die Prophylaxe chronischer Schmerzen spielt die Versorgung akuter Schmerzen eine große Rolle. Werden Schmerzen der Kinder in der Akutphase ausreichend behandelt, sinkt das Chronifizierungsrisiko. „Bei Behandlungen sollten Kinder und Jugendliche generell so wenig Schmerzen wie möglich erleben", unterstreicht ÖSG-Präsidentin OÄ Dr. Waltraud Stromer. Manche Diagnosen und Therapien kommen aber ohne Nadelstich nicht aus, etwa Blutentnahmen, Injektionen oder Impfungen. „Um den Kindern hier Schmerzen zu ersparen, kann vor dem Stich die Einstichstelle mit Lidocaincreme schmerzunempfindlich gemacht werden. Eine behaglich-beruhigende Positionierung lindert zusätzlich den Schmerz. Bei Säuglingen kann der Stich beim Stillen erfolgen, älteren Kindern sollte eine altersgerechte Ablenkung geboten werden. Wenn das alles nicht hilft, ist die Sedierung mit Lachgas eine mögliche Ausweichlösung", erklärt die Expertin.

\section{Kommunikation mit Kindern und Eltern wichtig}

Die Basis einer optimalen Schmerzprophylaxe ist eine offene und klare Kommunikation zwischen Ärzt*innen und Kindern. Die Ärzt*innen müssen darauf achten, den Kindern immer die Wahrheit zu sagen, eine kindgerechte Sprache zu verwenden und angstmachende Wörter zu vermeiden. Eltern bzw. Vertrauenspersonen müssen immer einbezogen werden. Eltern von Kindern mit chronischen Schmerzen sind durch die Situation häufig emotional stark belastet und neigen zu schmerzverstärkenden Reaktionen. „Kindergerechte Rahmenbedingungen, geschultes Personal und die Unterstützung durch die Eltern sind wesentliche Faktoren, um das Stresserleben von Kindern zu vermindern", sagt Dr. Platzer.

Kinder "resilient" zu machen, kann ebenfalls chronischen Schmerzen vorbeugen. Das bedeutet, dass Faktoren wie Selbstbewusstsein, positive Lebenshaltung und ein unterstützendes soziales Umfeld gefördert und das Verhalten und die Einstellung bei chronischen Schmerzen verändert werden. „Es verbessert die Lebensqualität, wenn es den Kindern trotz bestehender Schmerzen erleichtert wird, weiter an Tätigkeiten teilhaben zu können, die ihnen Freude bereiten“, erklärt Dr. Platzer. Der Alltag der Kinder mit Schule, Schlaf, sozialem Kontakt und Sport sollte daher normal weiterlaufen können. Vor allem aber müssen auch Risikofaktoren wie Angstgefühle und Stress, die Schmerz auslösen oder verstärken, möglichst bald erkannt und reduziert werden.

\section{Verbesserung der Versorgungssituation notwendig}

„Noch gibt es zu wenige schmerztherapeutische Angebote, die auf die speziellen Bedürfnisse für Kinder und Jugendliche ausgerichteten sind und gut miteinander vernetzt sind, wir haben hier großen Aufholbedarf", kritisiert Dr. Stromer. Allgemeinmediziner*innen hätten oft nicht die Zeit, um auf die psychosoziale Situation der jungen Schmerzpatient ${ }^{*}$ innen ausreichend einzugehen. In Zukunft könnten digitale Angebote die Face-to-Face-Versorgung hilfreich ergänzen.,Das ist vielversprechend, zumal Kinder und Jugendliche sehr medienaffin und -geübt sind“, schließt Dr. Platzer.

Bericht:Dr. Stefan Wolfinger

Quellen: KIGGS: Studie zur Gesundheit von Kindern und Jugendlichen in Deutschland. www.kiggs-studie.de; Zernikow B: Chronische Schmerzen bei Kindern und Jugendlichen. Die stille Epidemie. Schmerz 2021, 35:80-85; Platzer, M: Symposium "Gib dem Schmerz keine Chance aktuelle Empfehlungen zur Kinderschmerztherapie“ 24. September 2021, Villach (hybrid); Stromer W, Voitl P: Schmerztherapie bei Kindern und Jugendlichen. Schmerz Nachrichten 1/2021, 69-75

Hinweis des Verlags. Der Verlag bleibt in Hinblick auf geografische Zuordnungen und Gebietsbezeichnungen in veröffentlichten Karten und Institutsadressen neutral.

Schmerz Nachr 2022 $222: 13$

https://doi.org/10.1007/s44180-022-00010-5

Online publiziert: 24 . Februar 2022

(c) The Author(s), under exclusive licence to Springer-Verlag GmbH Austria, ein Teil von Springer Nature 2022 